\title{
Rottlerin inhibits cell growth, induces apoptosis and cell cycle arrest, and inhibits cell invasion in human hepatocellular carcinoma
}

\author{
JICHAN SHI ${ }^{1}$, HONGYE NING ${ }^{1}$, GUIQING HE ${ }^{1}$, YITONG HUANG ${ }^{2}$, \\ ZHENGXING WU $^{1}$, LINGLING JIN ${ }^{1}$ and XIANGAO JIANG ${ }^{1}$ \\ Departments of ${ }^{1}$ Infectious Disease and ${ }^{2}$ Gynecologic Oncology, Wenzhou Central Hospital, \\ Wenzhou, Zhejiang 325000, P.R. China
}

Received January 6, 2017; Accepted August 1, 2017

DOI: $10.3892 / \mathrm{mmr} .2017 .7924$

\begin{abstract}
Rottlerin, a polyphenolic compound, has been demonstrated to exhibit antitumor activity in various types of human cancer. Several studies have revealed that rottlerin exerts its anticancer function through PKC-dependent and independent pathways. The transcriptional co-activator with PDZ-binding motif (TAZ) oncopreotein is an important molecule in regulation of the Hippo pathway in human cancer. The present study investigated whether rottlerin has a tumor suppressive role via inhibiting the expression of TAZ, using cell viability assay, apoptosis and cell cycle analyses, western blot analysis and Tanswell invasion assay. The results demonstrated that rottlerin suppressed cell growth, triggered cell apoptosis and induced cell cycle arrest. In addition, rottlerin inhibited cell migration and invasion in hepatocellular carcinoma (HCC) cells. Mechanistically, the results demonstrated that rottlerin exerted its antitumor activity partly through the inhibition of TAZ. In addition, the depletion of TAZ led to inhibited cell growth and invasion, whereas the overexpression of TAZ enhanced cell growth and invasion in the HCC cells. Taken together, these findings indicated that the inhibition of TAZ by rottlerin may be a novel strategy for treating HCC.
\end{abstract}

\section{Introduction}

Hepatocellular carcinoma (HCC) is a life-threatening type of cancer, which is one of the common tumors in humans. It was predicted that there were 39,230 new cases of HCC with an HCC-associated mortality rate of 27,170 in 2016 in

Correspondence to: Dr Xiangao Jiang, Department of Infectious Disease, Wenzhou Central Hospital, 32 West Jiangbin Road, Wenzhou, Zhejiang 325000, P.R. China

E-mail: xiangaojiang@163.com

Key words: rottlerin, transcriptional co-activator with PDZ-binding motif, proliferation, apoptosis, invasion the United States (1). In contrast to the decreasing trends for other major cancer types, the mortality rate for HCC has increased. In China, 466,100 new cases of HCC were predicted and 422,100 patients were predicted to succumb to HCC-associated mortality in 2015 (2). Although several treatments, including surgery, radiation and chemotherapy, have been used to treat patients with HCC, the survival rate has not improved significantly (3). Therefore, the identification of novel agents is essential to improve the treatment effects in patients with HCC.

Rottlerin, also known as mallotoxin, is isolated from Mallotus phillippinensis (4). It has been reported that rottlerin inhibits tumorigenesis through the regulation of several mechanisms involving cells, including cell survival, apoptosis, autophagy and invasion (4). Rottlerin was initially identified as a potential protein kinase C (PKC) inhibitor 20 years ago (5). Several studies have confirmed that rottlerin inhibits cell proliferation and induces cell cycle arrest via the inhibition of protein kinase in several types of tumor in humans $(6,7)$. Subsequent studies have revealed that rottlerin exerts its tumor suppressor function via a PKC-independent pathway. For example, rottlerin was found to sensitize to tumor necrosis-related apoptosis-inducing ligand (TRAIL) -induced apoptosis through uncoupling of the mitochondria independently of PKC (8). Similarly, rottlerin was found to sensitize TRAIL-induced apoptosis via the suppression of cell division cycle $(\mathrm{Cdc}) 2$, survivin and X-linked inhibitor of apoptosis (XIAP) in glioma cells (9). Rottlerin has also been shown to suppress nuclear factor- $\kappa \mathrm{B}$ and cyclin D1 in breast cancer cells (10). Studies have also shown that rottlerin inhibits caspase-2, and induces autophagy and apoptotic cell death $(11,12)$. These findings indicate that rottlerin may regulate multiple genes to inhibit tumorigenesis.

The oncoprotein transcriptional co-activator with PDZ-binding motif (TAZ) has been identified as a key driver in the Hippo pathway; the Hippo signaling pathway is an essential regulator of organ size during developmental growth (13). Two transcription factors in the Hippo pathway, Yes-associated protein (YAP) and TAZ, have shown to possess oncogenic functions. The overexpression of TAZ was found to be significantly associated with poor overall survival in HCC 
and gastrointestinal cancer (14). The overexpression of TAZ is also associated with certain clinicopathologic characteristics, including tumor-node-metastasis stage, lymph node metastasis and tumor differentiation (14). Higher expression levels of TAZ have also been reported to indicate a poor prognosis in retinoblastoma (15). Therefore, TAZ offers potential as a therapeutic target in human cancer (16). The present study aimed to investigate whether rottlerin inhibits cell growth, migration and invasion, and whether it induces cell apoptosis and cell cycle arrest in HCC cells. In addition, the present study examined whether rottlerin affects the expression of TAZ in HCC cells. The results demonstrated that rottlerin suppressed cell growth, triggered cell apoptosis and induced cell cycle arrest. In addition, rottlerin inhibited cell migration and invasion of the HCC cells. Mechanistically, the results showed that rottlerin exerted its antitumor activity partly through the inhibition of TAZ. Taken together, these findings indicated that the inhibition of TAZ by rottlerin may be a useful approach for treating HCC.

\section{Materials and methods}

Cell culture and reagents. The human QGY-7703 cell line was purchased from the Cell Bank of Type Culture Collection of Chinese Academy of Sciences (Shanghai, China), and cultured in DMEM (Gibco; Thermo Fisher Scientific, Inc., Waltham, MA, USA) supplemented with $10 \%$ fetal bovine serum (Invitrogen; Thermo Fisher Scientific, Inc.) and 1\% penicillin-streptomycin in a $5 \% \mathrm{CO}_{2}$ atmosphere at $37^{\circ} \mathrm{C}$. Anti-TAZ antibody (1:1,000, sc-17130) was purchased from Santa Cruz Biotechnology, Inc. (Dallas, TX, USA). Secondary antibodies (anti-mouse HRP-linked antibody, \#7076, 1:4,000; anti-rabbit HRP-linked antibody, \#7074, 1:4,000) were purchased from Cell Signaling Technology, Inc. (Danvers, MA, USA). Monoclonal anti-tubulin antibody (1:3,000, T-3526), rottlerin (CAS no. R5648; $\geq 85 \%$ rottlerin) and Cell Titer Glo (CTG) were obtained from Sigma-Aldrich (Merck KGaA, Darmstadt, Germany). Rottlerin was dissolved in DMSO to produce a $30 \mathrm{mM}$ stock solution, and was added directly to the medium at different concentrations. Cells were treated with $0.1 \%$ DMSO as the control group.

Cell viability assay. The cells were seeded at a density of $8 \times 10^{3}$ cells/well in a 96-well plate for $24 \mathrm{~h}$ and treated with different concentrations $(1,2,3$ and $4 \mu \mathrm{M})$ of rottlerin in a humidified $\mathrm{CO}_{2}$ incubator at $37^{\circ} \mathrm{C}$. After 48 and $72 \mathrm{~h}, 20 \mu \mathrm{l}$ of CTG $(5 \mathrm{mg} / \mathrm{ml})$ solution was added to each well and incubated for $10 \mathrm{~min}$ at $37^{\circ} \mathrm{C}$. The reaction mixture was then measured on a microplate reader at $490 \mathrm{~nm}$ (17).

Analysis of cell apoptosis. Cells ( $3 \times 10^{5}$ cells/well) were cultured in a 6-well plate overnight and treated with the various concentrations of rottlerin for $48 \mathrm{~h}$. Following treatment, the cells were harvested and washed with PBS, resuspended ( $1 \times 10^{5}$ cells) in $500 \mu \mathrm{l}$ binding buffer with $5 \mu \mathrm{l}$ propidium iodide (PI) and $5 \mu$ l FITC-conjugated anti-Annexin V antibody. Apoptosis was analyzed on a flow cytometer (BD Biosciences, Franklin Lakes, NJ, USA) as described previously (18).

Cell cycle analysis. Exponentially growing cells $\left(2 \times 10^{5}\right.$ cells/well) were seeded in a 6 -well plate overnight and then treated with the different concentrations of rottlerin for $48 \mathrm{~h}$. After $48 \mathrm{~h}$, the cells were collected and washed with cold PBS. The cells were suspended with $70 \%$ cold alcohol and were maintained at $4^{\circ} \mathrm{C}$ overnight. Prior to analysis, the cells were washed with cold PBS, and resuspended at a density of $1 \times 10^{6}$ cells $/ \mathrm{ml}$ in PBS. The cells were incubated with $0.1 \mathrm{mg} / \mathrm{ml} \mathrm{RNase} \mathrm{I}$ and $50 \mathrm{mg} / \mathrm{ml} \mathrm{PI}$ at $37^{\circ} \mathrm{C}$ for $30 \mathrm{~min}$. Cell cycle was analyzed on a flow cytometer (BD Biosciences) as described previously (18).

Western blot analysis. The harvested cells were washed in PBS and lysed with protein lysis buffer containing $50 \mathrm{mmol} / 1$ Tris (pH 7.5), $100 \mathrm{mmol} / 1 \mathrm{NaCl}, 1 \mathrm{mmol} / 1$ EDTA, $0.5 \%$ NP40, $0.5 \%$ Triton X-100, $2.5 \mathrm{mmol} / \mathrm{l}$ sodium orthovanadate, $10 \mu \mathrm{l} / \mathrm{ml}$ protease inhibitor cocktail and $1 \mathrm{mmol} / \mathrm{l} \mathrm{PMSF}$. The concentrations of proteins were measured using a Bicinchoninic Acid Protein Assay kit (Thermo Fisher Scientific, Inc.). Equal quantities of protein samples $(30 \mu \mathrm{g})$ were separated by electrophoresis on a $10 \%$ sodium dodecyl sulphate-polyacrylamide gel and then transferred onto a polyvinylidene difluoride membrane. The membrane was then incubated with the primary antibodies at $4^{\circ} \mathrm{C}$ overnight. Following incubation, the membrane was washed with TBST three times and then incubated with the secondary antibody at room temperature for $1 \mathrm{~h}$. The expression of protein was detected using an electrochemiluminescence assay and were analyzed using ImageJ version 1.46r (National Institutes of Health, Bethesda, MD, USA).

Cell invasion analysis. A Transwell invasion assay was used to measure the invasive capacity of the HCC cells according to the manufacturer's protocol. HCC cells in serum-free medium containing rottlerin $(1,2$ or $3 \mu \mathrm{M})$ were seeded onto inserts in the upper chamber $\left(1 \times 10^{4}\right.$ cells/well $)$ in a 24 -well plate. The lower wells were filled with complete medium with the same concentration of rottlerin. Following incubation for $20 \mathrm{~h}$ in a humidified $\mathrm{CO}_{2}$ incubator at $37^{\circ} \mathrm{C}$, the cells in the upper chambers were removed using cotton buds. The cells on the lower surface of the chambers were stained with $4 \mu \mathrm{g} / \mathrm{ml}$ Calcein $\mathrm{AM}$ in PBS at $37^{\circ} \mathrm{C}$ for $1 \mathrm{~h}$. Images of these fluorescently labeled invasive cells were captured under a fluorescent microscope. The invaded cells on the membrane were stained with Wright's-Giemsa and images were captured (17).

Cell transfection. The cells were seeded into a 6-well plate and transfected with TAZ small interfering (si)RNA or control siRNA (A06001; Shanghai GenePharma Co., Ltd., Shanghai, China) using Lipofectamine 2000 according to the manufacturer's protocol. The TAZ siRNA sequences were as follows: Sense, 5'-GCAUCUUCGACAGUCUUCUTT-3' and antisense, 5'-AGAAGACUGUCGAAGAUGCTT-3'. Following transfection, the cells were subjected to the analyses described above.

Statistical analysis. All statistical analyses were performed using GraphPad Prism 5.0 (Graph Pad Software, Inc., La Jolla, CA, USA). Student's t-test was performed to evaluate statistical significance. The results are presented as the mean \pm standard deviation. $\mathrm{P}<0.05$ was considered to indicate a statistically significant difference. 

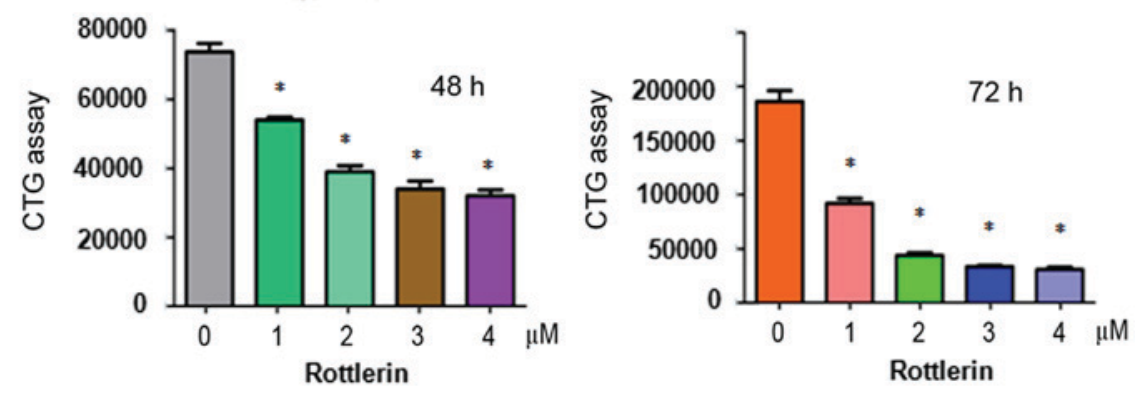

Figure 1. Effect of rottlerin on cell growth. Cell growth was detected using a CTG assay in QGY-7703 cells following rottlerin treatment. Data are presented as cell numbers. ${ }^{*} \mathrm{P}<0.05$, vs. control.
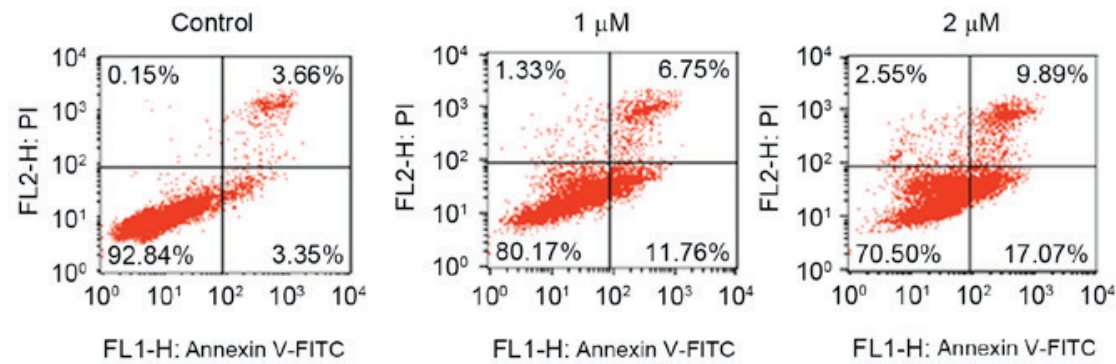

Figure 2. Effect of rottlerin on cell apoptosis. The apoptosis of cells treated with rottlerin for $48 \mathrm{~h}$ was determined using flow cytometry in QGY-7703 cells. PI, propidium iodide; FITC, fluorescein isothiocyanate.
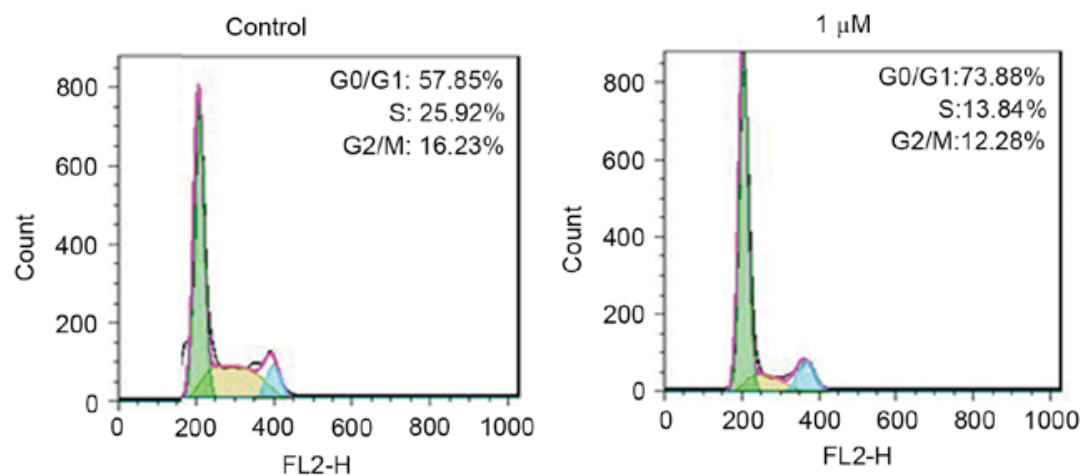

Figure 3. Effect of rottlerin on cell cycle. Cell cycle analysis was performed by PI staining and flow cytometry for QGY-7703 cells treated with rottlerin for $48 \mathrm{~h}$. Rottlerin induced cell cycle arrest in the hepatocellular carcinoma cells.

\section{Results}

Rottlerin inhibits cell proliferation. To determine whether rottlerin suppresses the proliferation of HCC cells, a CTG assay was performed to examine the viability of QGY-7703 cells treated with different concentrations of rottlerin for 48 and $72 \mathrm{~h}$. The results showed that rottlerin significantly inhibited cell proliferation in the two HCC cell lines (Fig. 1). The half maximal inhibitory concentration $\left(\mathrm{IC}_{50}\right)$, which is the concentration leading to $50 \%$ cell growth inhibition, was $\sim 1 \mu \mathrm{M}$ at $72 \mathrm{~h}$ for the QGY-7703 cells. These results suggested that the QGY-7703 cells were sensitive to rottlerin treatment. Therefore, a $1 \mu \mathrm{M}$ concentration of rottlerin was used for QGY-7703 cells in the subsequent experiments.

Rottlerin induces apoptosis. The present study also aimed to determine whether rottlerin enhances the apoptosis of HCC cells. A PI-FITC-Annexin assay was performed to measure the rates of apoptotic death of HCC cells following treatment with rottlerin for $48 \mathrm{~h}$. It was found that rottlerin triggered cell apoptosis in the HCC cells (Fig. 2). Specifically, cell apoptosis was increased from $7.0 \%$ in the control group to 18.5 and $26.96 \%$ in the 1 and $2 \mu \mathrm{M}$ rottlerin-treated QGY-7703 cell groups, respectively (Fig. 2). These findings indicated that rottlerin stimulated the apoptosis of HCC cells.

Rottlerin induces cell cycle arrest. To further define the role of rottlerin in cell growth inhibition, the present study measured cell cycle in the HCC cells following rottlerin treatment. Cell cycle analysis using PI staining and flow cytometry was performed in the HCC cells. The results revealed that rottlerin induced $\mathrm{G}_{0} / \mathrm{G}_{1}$ arrest in the QGY-7703 cells (Fig. 3). Treatment with $1 \mu \mathrm{M}$ rottlerin led to an increase in the percentage of $\mathrm{G}_{0} / \mathrm{G}_{1}$ cells from 57.85 to $73.88 \%$ in the QGY-7703 cells. These 



Figure 4. Rottlerin inhibits QGY-7703 cell invasion. (A) Inhibitory effect of rottlerin on hepatocellular carcinoma cell invasion was detected using a Transwell chamber assay. The invaded cells on the membrane were stained with Wright's-Giemsa and images were captured (magnification, x40). (B) Quantification of the results. "P $<0.05$ and ${ }^{* *} \mathrm{P}<0.01$, vs. control.
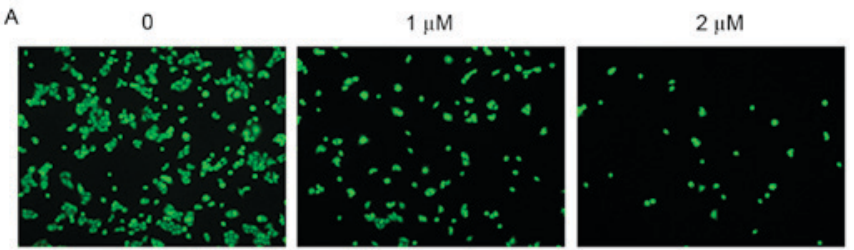

B



Figure 5. Rottlerin inhibits QGY-7703 cell invasion. (A) Inhibitory effect of rottlerin on cell invasion was measured using a Transwell chamber assay. The hepatocellular carcinoma cells in serum-free medium containing rottlerin were seeded into the upper chamber of inserts in a 24 -well plate. Lower wells were filled with complete medium containing the same concentration of rottlerin. After $20 \mathrm{~h}$, the cells on the lower surface of the chambers were stained with $4 \mu \mathrm{g} / \mathrm{ml} \mathrm{Calcein} \mathrm{AM} \mathrm{in} \mathrm{PBS} \mathrm{buffer} \mathrm{at} 37^{\circ} \mathrm{C}$ for $1 \mathrm{~h}$. Images of the fluorescently-labeled invasive cells were captured (magnification, x10) under a fluorescent microscope. (B) Relative number of invaded cells. " $\mathrm{P}<0.05$, vs. control.

results demonstrated that rottlerin induced cell cycle $\mathrm{G}_{0} / \mathrm{G}_{1}$ arrest.

Rottlerin inhibits cell invasion. Rottlerin has been reported to inhibit cell invasion in pancreatic cancer cells (17). To analyze whether rottlerin can inhibit the cell motility of HCC cells, the present study performed an invasion assay using Matrigel-coated membranes. The results showed that rottlerin treatment led to decreased penetration of the $\mathrm{HCC}$ cells through the Matrigel-coated membrane, compared


Figure 6. Rottlerin downregulates the protein expression levels of TAZ (A) Expression of TAZ was determined using western blot analysis in QGY-7703 cells following rottlerin treatment. (B) Semi-quantification of relative TAZ expression. " $\mathrm{P}<0.05$ and ${ }^{* *} \mathrm{P}<0.01$, vs. control. TAZ, transcriptional co-activator with PDZ-binding motif.

with that of the control cells (Fig. 4). In addition, the results showed that the numbers of fluorescently-labeled invasive cells were significantly reduced by rottlerin treatment in the HCC cells (Fig. 5). These results suggested that rottlerin inhibited cell invasion of the HCC cells.

Rottlerin decreases the expression of TAZ. It has been shown that TAZ exerts oncogenic functions in tumorigenesis (19). To determine whether rottlerin mediates antitumor activity via regulating the expression of TAZ in $\mathrm{HCC}$ cells, the present study measured the expression of TAZ in HCC cells treated with rottlerin using western blot analysis. The results from the western blot analysis showed that rottlerin significantly downregulated the expression of TAZ in the QGY-7703 cells (Fig. 6). This finding indicated that rottlerin exhibited anticancer activity at least partly due to the inhibition of TAZ in HCC cells.

Down-regulation of TAZ enhances rottlerin-induced inhibition of cell growth. To further confirm the role of TAZ in rottlerin-induced cell growth inhibition, the HCC cells were transfected with TAZ siRNA. It was found that TAZ siRNA decreased the expression of TAZ in QGY-7703 cells (Fig. 7A). The results of the CTG assay showed that the depletion of TAZ inhibited cell growth (Fig. 7B). It was also observed that the downregulation of TAZ inhibited cell invasion (Fig. 7C). Of note, cell growth was significantly inhibited by rottlerin combined with TAZ siRNA transfection (Fig. 7D). These results suggested that rottlerin suppressed cell growth partly due to the inhibition of TAZ in HCC cells.

\section{Discussion}

There is increasing evidence supporting the potential antitumor activity of rottlerin in human cancer. For example, rottlerin has been shown to induce Wnt co-receptor LRP6 degradation, and inhibit Wnt/ $\beta$-catenin and mammalian target of rapamycin (mTOR) $\mathrm{C} 1$ signaling in prostate and breast cancer cells (20). Kumar et al (21) reported that rottlerin induced autophagy and apoptosis via regulation of the phosphoinositide 3-kinase (PI3K)/Akt/mTOR signaling pathway in prostate and pancreatic cancer stem cells (22). Lim et al (23) found that rottlerin induced apoptosis via regulating extracellular signal regulated kinase (ERK) and p38 mitogen-activated 
A

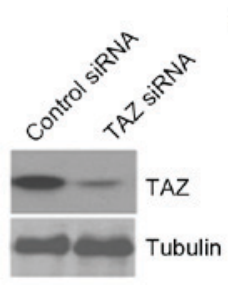

B



C

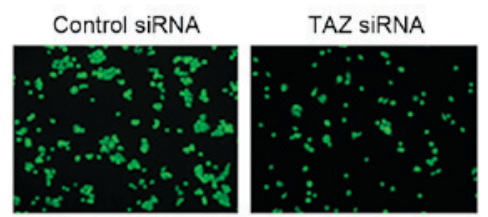

D

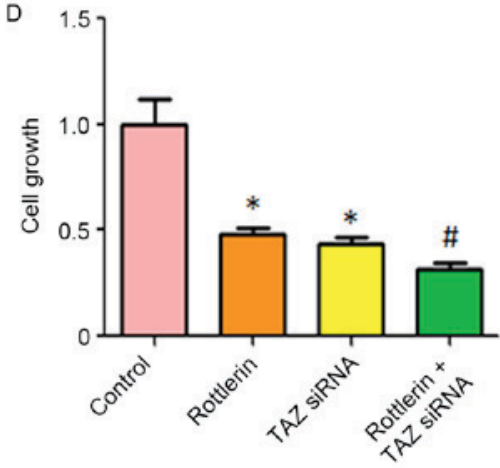

Figure 7. Effects of the downregulation of TAZ on cell growth and invasion. (A) Expression of TAZ was determined using western blot analysis in QGY-7703 cells transfected with TAZ siRNA. (B) A CTG assay was used to detect the effects of TAZ siRNA on HCC cell growth. Data are presented as relative cell growth (fold change). "P<0.05, vs. control. (C) Invasion assay for QGY-7703 cells transfected with TAZ siRNA (magnification, $x 10$ ). (D) CTG assay to examine the effects of TAZ siRNA in combination with rottlerin treatment on HCC proliferation. Data are presented as relative cell growth (fold change). ${ }^{*} \mathrm{P}<0.05$, vs. control; ${ }^{*} \mathrm{P}<0.05$, vs. rottlerin treatment or $\mathrm{TAZ}$ siRNA transfection. HCC, hepatocellular carcinoma; TAZ, transcriptional co-activator with PDZ-binding motif; siRNA, small interfering RNA.

protein kinase (MAPK) in colon carcinoma cells. Another study showed that rottlerin inhibited cell growth and invasion through the downregulation of Cdc20 in glioma cells (24). Rottlerin also exhibited antitumor effect via the inactivation of $\mathrm{S}$ phase kinase-associated protein 2 in pancreatic cancer cells and breast cancer cells $(17,18)$. These reports identified the mechanisms underlying rottlerin-mediated antitumor function. In the present study, it was found that rottlerin inhibited the expression of TAZ in HCC cells, suggesting that targeting TAZ by rottlerin is a potential approach for the treatment of HCC.

Previous studies have revealed that the overexpression of YAP/TAZ promotes the proliferation of cancer cells. Wang et al (25) reported that TAZ promoted cell growth and inhibited celastrol-induced cell apoptosis. In addition, the overexpression of TAZ was shown to enhance cell proliferation, migration and epithelial-mesenchymal transition (EMT) in ovarian cancer (26). In support of these findings, the present study observed that the overexpression of TAZ promoted cell growth and invasion, whereas the depletion of TAZ inhibited cell growth and invasion. TAZ has also been shown to promote EMT and support pancreatic cancer progression (27). In accordance, the knockdown of TAZ modifies cell sensitivity to EGFR inhibitors in triple-negative breast cancer cells (28). TAZ and YAP regulate pancreatic cancer initiation in mice via the direct upregulation of JAK-STAT3 signaling (29). Further investigation is required to examine the mechanism underlying TAZ-induced ovarian tumorigenesis.

As TAZ is a key oncoprotein, it is important to identify its inhibitors. Several groups have identified multiple inhibitors of TAZ. It has been reported that statins attenuate cell proliferative ability and induce apoptosis through TAZ in HCC cells (30), and statins have been shown to improve the prognosis of patients with HCC (30). Curcumin has been reported to downregulate the expression of TAZ in pancreatic cancer cells (31). In accordance, curcumin promotes the degradation of Krueppel-like factor 5 via the downregulation of YAP/TAZ in bladder cancer cells (32). Dasatinib, statins and pazopanid have been shown to inhibit the nuclear localization of TAZ via inducting the phosphorylation of TAZ (33). Pazopanib has also been shown to induce the proteasomal degradation of YAP/TAZ (33). The present study identified rottlerin as a potential inhibitor of TAZ in HCC. The present study confirmed that the rottlerin-induced inhibition of cell proliferation, induction of cell apoptosis and cell cycle arrest, and suppression of cell invasion and migration in HCC occurred partly through the downregulation of TAZ. Therefore, the inhibition of TAZ by rottlerin may be an effective approach for the treatment of HCC. Further investigations are required to investigate the functions of rottlerin in additional HCC cell lines and in $\mathrm{HCC}$ in vivo models.

\section{References}

1. Siegel RL, Miller KD and Jemal A: Cancer statistics, 2016. CA Cancer J Clin 66: 7-30, 2016.

2. Chen W, Zheng R, Baade PD, Zhang S, Zeng H, Bray F, Jemal A, Yu XQ and He J: Cancer statistics in China, 2015. CA Cancer J Clin 66: 115-132, 2016.

3. Kudo M, Trevisani F, Abou-Alfa GK and Rimassa L: Hepatocellular carcinoma: Therapeutic guidelines and medical treatment. Liver Cancer 6: 16-26, 2016.

4. Maioli E, Torricelli $\mathrm{C}$ and Valacchi G: Rottlerin and cancer: Novel evidence and mechanisms. Scientific World Journal 2012: 350826, 2012.

5. Gschwendt M, Müller HJ, Kielbassa K, Zang R, Kittstein W, Rincke $\mathrm{G}$ and Marks F: Rottlerin, a novel protein kinase inhibitor. Biochem Biophys Res Commun 199: 93-98, 1994.

6. Parmer TG, Ward MD and Hait WN: Effects of rottlerin, an inhibitor of calmodulin-dependent protein kinase III, on cellular proliferation, viability, and cell cycle distribution in malignant glioma cells. Cell Growth Differ 8: 327-334, 1997.

7. Ni H, Ergin M, Tibudan SS, Denning MF, Izban KF and Alkan S: Protein kinase $\mathrm{C}$-delta is commonly expressed in multiple myeloma cells and its downregulation by rottlerin causes apoptosis. Br J Haematol 121: 849-856, 2003.

8. Tillman DM, Izeradjene K, Szucs KS, Douglas L and Houghton JA: Rottlerin sensitizes colon carcinoma cells to tumor necrosis factor-related apoptosis-inducing ligand-induced apoptosis via uncoupling of the mitochondria independent of protein kinase C. Cancer Res 63: 5118-5125, 2003.

9. Kim EH, Kim SU and Choi KS: Rottlerin sensitizes glioma cells to TRAIL-induced apoptosis by inhibition of $\mathrm{Cdc} 2$ and the subsequent downregulation of survivin and XIAP. Oncogene 24: $838-849,2005$

10. Torricelli C, Fortino V, Capurro E, Valacchi G, Pacini A, Muscettola M, Soucek K and Maioli E: Rottlerin inhibits the nuclear factor kappaB/cyclin-D1 cascade in MCF-7 breast cancer cells. Life Sci 82: 638-643, 2008. 
11. Basu A, Adkins B and Basu C: Down-regulation of caspase- 2 by rottlerin via protein kinase $\mathrm{C}$-delta-independent pathway. Cancer Res 68: 2795-2802, 2008.

12. Song KS, Kim JS, Yun EJ, Kim YR, Seo KS, Park JH, Jung YJ, Park JI, Kweon GR, Yoon WH, et al: Rottlerin induces autophagy and apoptotic cell death through a PKC-delta-independent pathway in HT1080 human fibrosarcoma cells: The protective role of autophagy in apoptosis. Autophagy 4: 650-658, 2008.

13. Barron DA and Kagey JD: The role of the Hippo pathway in human disease and tumorigenesis. Clin Transl Med 3: 25, 2014.

14. Feng J, Ren P, Gou J and Li Z: Prognostic significance of TAZ expression in various cancers: A meta-analysis. Onco Targets Ther 9: 5235-5244, 2016.

15. Zhang Y, Xue C, Cui H and Huang Z: High expression of TAZ indicates a poor prognosis in retinoblastoma. Diagn Pathol 10: $187,2015$.

16. Zanconato F, Battilana G, Cordenonsi M and Piccolo S: YAP/TAZ as therapeutic targets in cancer. Curr Opin Pharmacol 29: 26-33, 2016.

17. Su J, Wang L, Yin X, Zhao Z, Hou Y, Ye X, Zhou X and Wang Z Rottlerin exhibits anti-cancer effect through inactivation of $S$ phase kinase-associated protein 2 in pancreatic cancer cells. Am J Cancer Res 6: 2178-2191, 2016.

18. Yin X, Zhang Y, Su J, Hou Y, Wang L, Ye X, Zhao Z, Zhou X, Li Y and Wang Z: Rottlerin exerts its anti-tumor activity through inhibition of Skp2 in breast cancer cells. Oncotarget 7: 66512-66524, 2016

19. Zanconato F, Cordenonsi $\mathrm{M}$ and Piccolo S: YAP/TAZ at the roots of cancer. Cancer Cell 29: 783-803, 2016.

20. Lu W, Lin C and Li Y: Rottlerin induces Wnt co-receptor LRP6 degradation and suppresses both Wnt/ $\beta$-catenin and mTORC1 signaling in prostate and breast cancer cells. Cell Signal 26: $1303-1309,2014$

21. Kumar D, Shankar S and Srivastava RK: Rottlerin induces autophagy and apoptosis in prostate cancer stem cells via PI3K/Akt/mTOR signaling pathway. Cancer Lett 343: 179-189, 2014.

22. Singh BN, Kumar D, Shankar S and Srivastava RK: Rottlerin induces autophagy which leads to apoptotic cell death through inhibition of PI3K/Akt/mTOR pathway in human pancreatic cancer stem cells. Biochem Pharmacol 84: 1154-1163, 2012.

23. Lim JH, Woo SM, Min KJ, Park EJ, Jang JH, Seo BR, Iqbal T, Lee TJ, Kim SH, Choi YH and Kwon TK: Rottlerin induces apoptosis of HT29 colon carcinoma cells through NAG-1 upregulation via an ERK and p38 MAPK-dependent and PKC $\delta$-independent mechanism. Chem Biol Interact 197: 1-7, 2012.
24. Wang L, Ye X, Cai X, Su J, Ma R, Yin X, Zhou X, Li H and Wang Z: Curcumin suppresses cell growth and invasion and induces apoptosis by down-regulation of Skp2 pathway in glioma cells. Oncotarget 6: 18027-18037, 2015.

25. Wang S, Ma K, Chen L, Zhu H, Liang S, Liu M and Xu N: TAZ promotes cell growth and inhibits Celastrol-induced cell apoptosis. Biosci Rep 36: pii: e00386, 2016.

26. Chen G, Xie J, Huang P and Yang Z: Overexpression of TAZ promotes cell proliferation, migration and epithelial-mesenchymal transition in ovarian cancer. Oncol Lett 12: 1821-1825, 2016.

27. Xie D, Cui J, Xia T, Jia Z, Wang L, Wei W, Zhu A, Gao Y, Xie K and Quan M: Hippo transducer TAZ promotes epithelial mesenchymal transition and supports pancreatic cancer progression. Oncotarget 6: 35949-35963, 2015.

28. Guo L, Zheng J, Zhang J, Wang H, Shao G and Teng L: Knockdown of TAZ modifies triple-negative breast cancer cell sensitivity to EGFR inhibitors by regulating YAP expression. Oncol Rep 36: 729-736, 2016.

29. Gruber R, Panayiotou R, Nye E, Spencer-Dene B, Stamp G and Behrens A: YAP1 and TAZ control pancreatic cancer initiation in mice by direct up-regulation of JAK-STAT3 signaling. Gastroenterology 151: 526-539, 2016.

30. Higashi T, Hayashi H, Kitano Y, Yamamura K, Kaida T, Arima K, Taki K, Nakagawa S, Okabe H, Nitta H, et al: Statin attenuates cell proliferative ability via TAZ (WWTR1) in hepatocellular carcinoma. Med Oncol 33: 123, 2016.

31. Zhou X, Su J, Feng S, Wang L, Yin X, Yan J and Wang Z: Antitumor activity of curcumin is involved in down-regulation of YAP/TAZ expression in pancreatic cancer cells. Oncotarget 7: 79076-79088, 2016.

32. Gao Y, Shi Q, Xu S, Du C, Liang L, Wu K, Wang K, Wang X, Chang LS, He D and Guo P: Curcumin promotes KLF5 proteasome degradation through downregulating YAP/TAZ in bladder cancer cells. Int J Mol Sci 15: 15173-15187, 2014.

33. Oku Y, Nishiya N, Shito T, Yamamoto R, Yamamoto Y, Oyama C and Uehara Y: Small molecules inhibiting the nuclear localization of YAP/TAZ for chemotherapeutics and chemosensitizers against breast cancers. FEBS Open Bio 5: 542-549, 2015. 